\title{
Oncological Outcomes of Bilateral Testicular Germ Cell Tumors and Evaluation of Prognostic Risk Factors
}

\author{
๑ İsmail Selvi ${ }^{1}$, ๑ Ali ìnsan Arık², ๑ Mehmet Sinan Başay² ${ }^{2}$ ๑ Halil Başar² \\ ${ }^{1}$ Başakşehir Çam and Sakura City Hospital, Clinic of Urology, Istanbul, Turkey \\ 2University of Health Sciences Turkey, Dr. Abdurrahman Yurtaslan Ankara Oncology Training and Research Hospital, Clinic of Urology, Ankara, Turkey
}

\begin{abstract}
Objective: The incidence of bilateral testicular germ cell tumours (TGCT) is low and constitutes $0.5 \%-7 \%$ of all testicular tumours. We aimed to evaluate the clinical and pathological features of unilateral and bilateral TGCT, as well as prognostic factors in bilateral cases that may have an impact on oncological outcomes. Materials and Methods: Bilateral TGCT were detected in $10(11.4 \%)$ of 87 patients between January 2010 and July 2016. Patients with 68 unilateral and 10 bilateral tumours (4 synchronous, 6 metachronous) had completely accessible data. We retrospectively evaluated their clinical-pathological data and postoperative follow-up results.

Results: Four patients with bilateral synchronous tumours had a seminoma and three (75\%) of them had a stage III disease. At a median follow-up of 31.50 (29-37) months, local recurrence, distant metastasis and death were observed in two patients with stage III disease. No recurrence or metastasis was seen in six patients with unilateral TGCT at 33 (24-50) months of follow-up, but metachronous tumours occurred in the contralateral testicles. At a median follow-up of 25 (11-39) months after metachronous tumour development, local recurrence, distant metastasis and death were observed in the contralateral testis of patients with stage III disease. There was no significant difference in bilateral and unilateral cases for disease-free survival, progression-free survival (PFS) and overall survival (OS). PFS and OS were significantly shorter $(p=0.039)$ in bilateral synchronous tumours than in metachronous tumours. Moreover, stage III disease was more common $(75 \%$ vs $33.3 \%$ ) in synchronous tumours. Family history (OR: 6.556, $p=0.035)$, testicular dysgenesis syndrome (OR: $3.876, p=0.031)$, disorders of semen parameters (OR: 2.879, $\mathrm{p}=0.037$ ), undescended testis (OR: 2.561, $\mathrm{p}=0.026)$, monocyte/lymphocyte ratio $>0.31$ [odds rotio (OR): $2.234, \mathrm{p}=0.022$ ], testicular microlithiasis (OR: 2.015, $p=0.015)$ and neutrophil/lymphocyte ratio $>3.23$ (OR: 1.348, $p=0.025)$ increased the risk of contralateral tumour development.

Conclusion: Bilateral synchronous tumours are detected at a more advanced stage and have lower PFS and OS durations, but survival rates are similar to those of unilateral tumours. Long-term follow-up is necessary for patients with unilateral TGCT having certain risk factors due to the possibility of metachronous tumour development in the contralateral testis.

Keywords: Bilateral, metachronous, synchronous, testicular dysgenesis syndrome, testicular germ cell tumour
\end{abstract}

\section{Introduction}

Although testicular germ cell tumor (TGCT) constitutes $1 \%$ of all male malignancies and $5 \%$ of urological tumors, it is the most common solid tumor detected in men aged 15-44 years (1). In the treatment of TGCT, which accounts for more than $95 \%$ of all testicular malignancies, the standard first approach is radical orchiectomy, and as a result of the developments in adjuvant chemotherapy (CT), high rates of cure can be achieved today (2).

Well-known risk factors in the etiology are testicular dysgenesis syndrome (TDS) components (undescended testis, hypospadias, decreased spermatogenesis), familial TGCT history in firstdegree relatives, a history of tumor in the contralateral testicle, and the presence of intratubular germ cell neoplasia (ITGCN) (3). Bilateral TGCT accounts for $0.5-7 \%$ of all testicular tumors $(1,4)$. Synchronous (simultaneous bilateral) tumor is observed in approximately $35 \%$ of these patients, and metachronous (second contralateral) tumor is seen in 65\% (5).

In general, although it is known that synchronous tumors are seen at more advanced stages and survival rates are lower than metachronous tumors, our knowledge about epidemiological and clinicopathological features and treatment strategies of bilateral TGCT is limited (1). Therefore, we aimed to evaluate the prognostic factors that might affect oncological outcomes in patients with bilateral TGCT by examining the clinical and pathological features of patients diagnosed as having bilateral or unilateral TGCT in our clinic. 


\section{Materials and Methods}

All procedures in our study were conducted in accordance with the ethical standards of the institutional and national research committee including human participants and the principles of the Helsinki Declaration, and since it was a retrospective study, no ethics committee approval was made. Each patient was informed before the surgery that the demographic, clinical, pathological and oncological data of the patients could be used in various oncological studies to be performed in the clinic without specifying the patient names and identity information, and the data of the patients who did not consent was not used in this study.

Pathological findings of 99 patients who underwent radical orchiectomy with a pre-diagnosis of testicular tumor between January 2010 and July 2016 were evaluated retrospectively. The patients of whom pathological examination of orchiectomy specimens showed paratesticular sarcoma $(n=5)$, epidermoid cyst $(n=1)$, inflammatory myofibroblastic tumor $(n=1)$, benign cystic teratoma $(n=2)$, benign leydig cell tumor $(n=1)$, paratesticular fibroma $(n=1)$ and non-Hodgkin lymphoma $(n=1)$ were excluded from the study, and the data of 87 patients with TGCT were evaluated.

Demographic data, histopathological tumor subtypes, clinical tumor stage, tumor localization, tumor size, expression of serum tumor markers (alpha-fetoprotein, beta human chorionic gonadotropin, lactate dehydrogenase), serum hemogram parameters [(neutrophil/lymphocyte ratio (NLR)], monocyte/ lymphocyte ratio (MLR), platelet/lymphocyte ratio (PLR), mean platelet volume, red cell distribution width], prognostic factors of tumor in pathology samples, follow-up time after orchiectomy, local recurrence, distant metastasis and overall mortality rates of the patients were noted. Family history, the presence of undescended testis, hypospadias or semen parameter disorder (the presence of any disorder in terms of number, motility, morphology or vitality in semen parameters), presence of atrophic testis (testicular volume $<12 \mathrm{~mL}$ ), and the status of microlithiasis in preoperative ultrasonographic examination were also recorded by scanning patient files. Seventy eight patients with TGCT who could be reached were included.

Clinical tumor stages were evaluated according to the 2009 tumor-node-metastasis classification. While early stage tumors consisted of stage IA and IB; advanced stage tumors included stage IS, IIA/IIB/IIC and IIIA/IIIB/IIIC. Histological tumor subtypes were evaluated according to the World Health Organization's classification. According to the European Association of Urology (EAU) 2020 guidelines, for stage I seminoma, presence of rete testicular involvement and tumor size greater than $4 \mathrm{~cm}$; for stage I non-seminoma, the presence of lymphovascular invasion, embryonal carcinoma rate over $50 \%$ and proliferation index over $70 \%$ were evaluated as pathological prognostic risk factors (3).

The definition of TDS includes the presence of at least two of undescended testicles, hypospadias, decreased spermatogenesis or TGCT (6). Since all patients in our study had TGCT, we classified the patients as having TDS due to whether having at least one of undescended testis, hypospadias or semen parameters disorder.
Patients were divided into two groups without randomization. Group I included patients with bilateral TCHT $(n=10)$, group II with unilateral TGCT $(n=68)$. Both groups were compared in terms of demographic and clinical data, prognostic risk factors, serum hemogram parameters and oncological results. Patients with bilateral TGCT were also evaluated by dividing them into two subgroups as synchronous $(n=4)$ and metachronous $(n=6)$.

\section{Statistical Analysis}

After evaluating the normality status with the KolmogorovSmirnov and Shapiro-Wilk tests, when comparing the differences between the two groups, Mann-Whitney $U$ test was used for continuous variables that did not show normal distribution. Pearson chi-square analysis or Fisher's Exact test was used for categorical variables. Kaplan-Meier method was used for the analysis of disease-free survival (DFS), progressionfree survival (PFS) and overall survival (OS), while differences between patient groups were evaluated using the log rank test. Logistic regression analysis was used to determine prognostic factors that may affect contralateral tumor development in the follow-up of patients with unilateral tumors at the time of initial diagnosis. Receiver operating characteristic analysis could not be performed to determine the threshold value for serum hemogram parameters due to the small sample size. Instead, the median values of all 78 patients included in the study were taken as threshold values and included in univariate and multivariate models in logistic regression analysis. Analyzes were performed using IBM SPSS Statistics 21 (IBM, Armonk, NY USA) software. Values of $p<0.05$ were considered statistically significant.

\section{Results}

\section{Patient Population and General Characteristics}

Bilateral tumors were detected in $10(11.4 \%)$ of 87 patients who were diagnosed as having TGCT after radical orchiectomy between January 2010 and July 2016. Four (40\%) of them had synchronous tumors and six $(60 \%)$ had metachronous tumors. Since the data were fully available, the median age of 78 patients included in the study was 31 and during a median of 57.50 (minimum $=6$ - maximum $=106)$ months follow-up, $15(19.2 \%)$ developed local recurrence, 17 (21.8\%) developed distant metastasis, and 16 (20.5\%) cancer-related death was observed. Distant metastases were detected in the lung in 8 patients, liver in 4 patients, and non-regional lymph nodes in 5 patients. Demographic, pathological, clinical data and oncological results of the patients are shown in Tables 1 and 2.

\section{Synchronous Tumors}

The median age at diagnosis was 33 (24-42) in 4 patients with bilateral synchronous TGCT. Two of the patients had a history of undescended testis, 1 had a history of hypospadias, 2 had a defect in semen parameters, 1 had atrophic testis, and 2 had testicular microlithiasis. Two patients were determined to be suitable for the TDS definition. Increased tumor markers were found in $3(75 \%)$ patients, while tumor histopathology was found as pure seminoma on both sides in all patients. After clinical staging, it was determined that one of the patients had 


\begin{tabular}{|c|c|c|c|c|}
\hline Parameters & $\begin{array}{l}\text { Group I } \\
(\text { bilateral TGCT) } \\
(n=10)\end{array}$ & $\begin{array}{l}\text { Group II } \\
\text { (unilateral TGCT) } \\
(n=68)\end{array}$ & $\begin{array}{l}\text { Total } \\
(n=78)\end{array}$ & p-value \\
\hline $\begin{array}{l}\text { Age } \\
\text { Median (25.-75. percentiles) }\end{array}$ & $27.00(24.75-36.00)$ & $31.00(25.25-41.00)$ & $31.00(25.00-39.50)$ & $\dagger 0.342$ \\
\hline $\begin{array}{l}\text { **Tumor size }(\mathrm{cm}) \\
\text { Median }(25 .-75 . \text { percentiles })\end{array}$ & $4.80(3.45-7.62)$ & $4.00(2.50-6.40)$ & $4.15(2.57-6.55)$ & $\dagger 0.282$ \\
\hline \multicolumn{5}{|l|}{ Tumor side (n, \%) } \\
\hline -Left & $0(0.0)$ & $25(36.8)$ & $25(32.1)$ & \multirow{3}{*}{$\stackrel{\ddagger}{\ddagger}<0.001^{*}$} \\
\hline -Right & $0(0.0)$ & $43(63.2)$ & $43(55.1)$ & \\
\hline -Bilateral & $10(100.0)$ & $0(0.0)$ & $10(12.8)$ & \\
\hline \multicolumn{5}{|c|}{ Histopathologic subtype (n, \%) } \\
\hline -Seminoma & $6(60.0)$ & $35(51.5)$ & $41(52.6)$ & \multirow{3}{*}{$\ddagger 0.877$} \\
\hline -Non-seminoma & $3(30.0)$ & $24(35.3)$ & $27(34.6)$ & \\
\hline -Mixed type & $1(10.0)$ & $9(13.2)$ & $10(12.8)$ & \\
\hline $\begin{array}{l}\text { AFP }(n g / m L) \\
\text { Median (25.-75. percentiles) }\end{array}$ & $4.35(2.07-82.02)$ & $6.15(2.15-76.06)$ & $5.60(2.17-72.68)$ & $\dagger 0.864$ \\
\hline $\begin{array}{l}\beta \text {-hCG (mlU/mL) } \\
\text { Median (25.-75. percentiles) }\end{array}$ & $5.00(1.75-370.00)$ & $17.95(2.55-114.00)$ & $12.45(2.50-124.00)$ & $\dagger 0.899$ \\
\hline $\begin{array}{l}\mathrm{LDH}(\mathrm{U} / \mathrm{L}) \\
\text { Median (25.-75. percentiles) }\end{array}$ & $\begin{array}{l}608.50 \\
(230.00-1090.75)\end{array}$ & $\begin{array}{l}308.50 \\
(187.75-717.00)\end{array}$ & $\begin{array}{l}309.00 \\
(201.35-784.50)\end{array}$ & $\dagger 0.145$ \\
\hline \multicolumn{5}{|l|}{ Tumor stage } \\
\hline-1 & $5(50.0)$ & $38(55.9)$ & $43(55.1)$ & \multirow{3}{*}{$\S 0.455$} \\
\hline-11 & $0(0.0)$ & $8(11.8)$ & $8(10.3)$ & \\
\hline-111 & $5(50.0)$ & $22(32.4)$ & $27(34.6)$ & \\
\hline \multicolumn{5}{|l|}{$\operatorname{ITGCN}(n, \%)$} \\
\hline Yes & $8(80.0)$ & $30(44.1)$ & $38(48.7)$ & \multirow{2}{*}{$\ddagger 0.034^{*}$} \\
\hline No & $2(20.0)$ & $38(55.9)$ & $40(51.3)$ & \\
\hline \multicolumn{5}{|l|}{ Rete testis invasion $(n, \%)$} \\
\hline Yes & $2(20.0)$ & $16(23.5)$ & $18(23.1)$ & \multirow{2}{*}{$\S 0.582$} \\
\hline No & $8(80.0)$ & $52(76.5)$ & $60(76.9)$ & \\
\hline \multicolumn{5}{|l|}{ Tumor diameter $>4 \mathrm{~cm}(\mathrm{n}, \%)$} \\
\hline Yes & $7(70.0)$ & $33(48.5)$ & $40(51.3)$ & \multirow{2}{*}{$\S 0.312$} \\
\hline No & $3(30.0)$ & $35(51.5)$ & $38(48.7)$ & \\
\hline \multicolumn{5}{|c|}{ Lymphovascular invasion (n, \%) } \\
\hline Yes & $4(40.0)$ & $23(33.8)$ & $27(34.6)$ & \multirow{2}{*}{$\S_{0.478}$} \\
\hline No & $6(60.0)$ & $45(66.2)$ & $51(65.4)$ & \\
\hline \multicolumn{5}{|c|}{ Embryonal carcinoma rate $>50 \%(n, \%)$} \\
\hline Yes & $1(10.0)$ & $18(26.5)$ & $19(24.4)$ & \multirow{2}{*}{$\S 0.436$} \\
\hline No & $9(90.0)$ & $50(73.5)$ & $59(75.6)$ & \\
\hline \multicolumn{5}{|l|}{ Proliferation rate $>70 \%(n, \%)$} \\
\hline Yes & $1(10.0)$ & $1(1.5)$ & $2(2.6)$ & \multirow{2}{*}{$\S_{0.241}$} \\
\hline No & $9(90.0)$ & $67(98.5)$ & $76(97.4)$ & \\
\hline \multicolumn{5}{|c|}{ 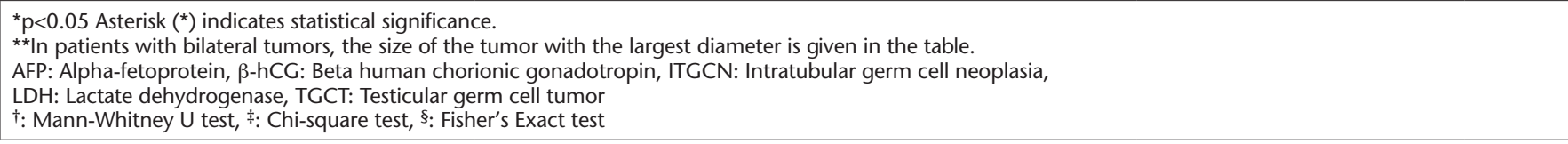 } \\
\hline
\end{tabular}

stage I disease and the other 3 had stage III disease. At a median follow-up of 31.50 (29-37) months, local recurrence, distant metastasis and death were observed in 2 patients with stage III disease (Tables 3 and 4). 


\begin{tabular}{|c|c|c|c|c|}
\hline Parameters & $\begin{array}{l}\text { Group I } \\
\text { (bilateral TGCT) } \\
(\mathrm{n}=10)\end{array}$ & $\begin{array}{l}\text { Group II } \\
\text { (unilateral TGCT) } \\
(\mathrm{n}=68)\end{array}$ & $\begin{array}{l}\text { Total } \\
(\mathrm{n}=78)\end{array}$ & p-value \\
\hline \multicolumn{5}{|l|}{ Undescended testis (n, \%) } \\
\hline Yes & $4(40.0)$ & $8(11.8)$ & $12(15.4)$ & \multirow{2}{*}{$\S 0.042^{\star}$} \\
\hline No & $6(60.0)$ & $60(88.2)$ & $66(84.6)$ & \\
\hline \multicolumn{5}{|l|}{ Semen parameter disorders $(n, \%)$} \\
\hline Yes & $4(40.0)$ & $7(10.3)$ & $11(14.1)$ & \multirow{2}{*}{$\S 0.030^{*}$} \\
\hline No & $6(60.0)$ & $61(89.7)$ & $67(85.9)$ & \\
\hline \multicolumn{5}{|l|}{ Hypospadias (n, \%) } \\
\hline Yes & $2(20.0)$ & $1(1.5)$ & $3(3.8)$ & \multirow{2}{*}{$\S 0.042^{*}$} \\
\hline No & $8(80.0)$ & $67(98.5)$ & $75(96.2)$ & \\
\hline \multicolumn{5}{|l|}{ Atrophic testis (n, \%) } \\
\hline Yes & $1(10.0)$ & $4(5.9)$ & $5(6.4)$ & \multirow{2}{*}{$\S 0.506$} \\
\hline No & $9(90.0)$ & $64(94.1)$ & $73(93.6)$ & \\
\hline \multicolumn{5}{|l|}{ Testicular microlithiasis (n, \%) } \\
\hline Yes & $5(50.0)$ & $14(20.6)$ & $19(24.4)$ & \multirow{2}{*}{$\ddagger 0.043^{*}$} \\
\hline No & $5(50.0)$ & $54(79.4)$ & $59(75.6)$ & \\
\hline \multicolumn{5}{|l|}{ Presence of TDS (n, \%) } \\
\hline Yes & $5(50.0)$ & $12(17.6)$ & $17(21.8)$ & \multirow{2}{*}{$\S 0.035^{*}$} \\
\hline No & $5(50.0)$ & $56(82.4)$ & $61(78.2)$ & \\
\hline \multicolumn{5}{|l|}{ Family history of TGCT (n, \%) } \\
\hline Yes & $4(40.0)$ & $9(13.2)$ & $13(16.7)$ & \multirow{2}{*}{$\ddagger 0.034^{*}$} \\
\hline No & $6(60.0)$ & $59(86.8)$ & $65(83.3)$ & \\
\hline Neutrophil/lymphocyte ratio & $5.34(4.33-7.45)$ & $2.76(1.80-4.42)$ & $3.23(2.09-4.58)$ & $\dagger 0.001^{*}$ \\
\hline Monocyte/lymphocyte ratio & $0.38(0.33-0.49)$ & $0.27(0.18-0.37)$ & $0.31(0.19-0.38)$ & $\dagger 0.006^{*}$ \\
\hline Platelet/lymphocyte ratio & $190.34(169.05-248.76)$ & $142.69(98.16-197.48)$ & $150.01(107.48-207.86)$ & $\dagger 0.013^{*}$ \\
\hline Mean platelet volume (fL) & $4.29(3.63-6.04)$ & $5.20(3.72-7.13)$ & $5.08(3.74-6.86)$ & †0.424 \\
\hline Red blood cell distribution width (fL) & $14.75(13.57-15.37)$ & $13.90(12.72-14.67)$ & $13.90(12.87-14.80)$ & $\dagger 0.081$ \\
\hline $\begin{array}{l}\text { Follow-up time [median (minimim- } \\
\text { maximum), months] }\end{array}$ & $49.50(29-69)$ & $60.50(6-106)$ & $57.50(6-106)$ & $\dagger 0.313$ \\
\hline Local recurrence rate $(n, \%)$ & $2(20.0)$ & $13(19.1)$ & $15(19.2)$ & $\S_{0.616}$ \\
\hline Distant metastasis rate $(\mathrm{n}, \%)$ & $3(30.0)$ & $14(20.6)$ & $17(21.8)$ & $\S 0.376$ \\
\hline Total mortality rate $(\mathrm{n}, \%)$ & $3(30.0)$ & $13(19.1)$ & $16(20.5)$ & $\S_{0.420}$ \\
\hline
\end{tabular}

\section{Metachronous Tumors}

In 6 patients with bilateral metachronous TGCT, the median age at diagnosis was 26 (20-35) when the first tumor was detected, and 29.5 (22-37) when the contralateral second tumor was detected. Two of the patients had a history of undescended testis, one had a history of hypospadias, 2 had a defect in semen parameters, and 3 had testicular microlithiasis. Three patients were consistent with the TDS definition. During the first tumor diagnosis, elevation in tumor markers was detected in 5 (83.3\%) patients, while tumor histopathology was determined as pure seminoma in 2 patients, pure non-seminoma in 3 patients, and mixed type TGCT in one patient. In this subgroup, where 4 patients had stage I disease and 2 patients had stage III disease, no recurrence or metastasis was observed at a median follow-up of 33 (24-50) months until the contralateral tumor developed (Tables 3 and 4).

When the contralateral second testicular tumor was detected, tumor markers were elevated in $3(50 \%)$ patients, while tumor histopathology was determined as pure seminoma in 4 patients and pure non-seminoma in 2 patients. Although the first tumor was not seminoma in 2 patients, it was observed that the contralateral tumor was seminoma. No significant difference was found in terms of initial tumor size, and contralateral tumor size developed at follow-up [4.75 (3-8) cm vs $4.35(3.1-6.1) \mathrm{cm}$, $\mathrm{p}=0.699$ ]. Unlike the initial tumor staging, stage III contralateral tumor was observed in only one patient and stage I contralateral 


\begin{tabular}{|c|c|c|c|c|}
\hline Parameters & $\begin{array}{l}\text { Synchronous tumors } \\
(\mathrm{n}=4)\end{array}$ & $\begin{array}{l}\text { Metachronous tumors } \\
(n=6)\end{array}$ & $\begin{array}{l}\text { Total } \\
(n=10)\end{array}$ & p-value \\
\hline $\begin{array}{l}\text { Age } \\
\text { Median (25.-75. percentiles) }\end{array}$ & $33.00(24.75-41.25)$ & $26.00(23.75-33.50)$ & $27.00(24.75-36.00)$ & $\dagger 0.334$ \\
\hline $\begin{array}{l}{ }^{* *} \text { Tumor size }(\mathrm{cm}) \\
\text { Median }(25 .-75 . \text { percentiles })\end{array}$ & $5.50(2.40-7.37)$ & $4.75(3.45-7.77)$ & $4.80(3.45-7.62)$ & $\dagger 0.831$ \\
\hline \multicolumn{5}{|c|}{ Histopathologic subtype (n, \%) } \\
\hline -Seminoma & $4(100.0)$ & $2(33.3)$ & $6(60.0)$ & \multirow{3}{*}{$\ddagger 0.035^{\star}$} \\
\hline -Non-seminoma & $0(0.0)$ & $3(50.0)$ & $3(30.0)$ & \\
\hline -Mixed type & $0(0.0)$ & $1(16.7)$ & $1(10.0)$ & \\
\hline $\begin{array}{l}\text { AFP }(\mathrm{ng} / \mathrm{mL}) \\
\text { Median (25.-75. percentiles) }\end{array}$ & $4.10(1.92-54.50)$ & $33.00(2.07-430.62)$ & $4.35(2.07-82.02)$ & $\dagger 0.522$ \\
\hline $\begin{array}{l}\beta \text {-hCG }(\mathrm{mlU} / \mathrm{mL}) \\
\text { Median (25.-75. percentiles) }\end{array}$ & $1.50(0.25-120.50)$ & $71.40(4.27-279.00)$ & $5.00(1.75-370.00)$ & $\dagger 0.088$ \\
\hline $\begin{array}{l}\mathrm{LDH}(\mathrm{U} / \mathrm{L}) \\
\text { Median (25.-75. percentiles) }\end{array}$ & $\begin{array}{l}1159.00 \\
(407.75-2304.25)\end{array}$ & $\begin{array}{l}408.00 \\
(230.00-880.00)\end{array}$ & $\begin{array}{l}608.50 \\
(230.00-1090.75)\end{array}$ & $\dagger 0.394$ \\
\hline \multicolumn{5}{|l|}{ Tumor stage } \\
\hline - & $1(25.0)$ & $4(66.7)$ & $5(50.0)$ & \multirow{3}{*}{$\ddagger 0.197$} \\
\hline-11 & $0(0.0)$ & $0(0.0)$ & $0(0.0)$ & \\
\hline-111 & $3(75.0)$ & $2(33.3)$ & $5(50.0)$ & \\
\hline \multicolumn{5}{|l|}{$\operatorname{ITGCN~(n,~\% )~}$} \\
\hline Yes & $4(100.0)$ & $4(66.7)$ & $8(80.0)$ & \multirow{2}{*}{$\$ 0.333$} \\
\hline No & $0(0.0)$ & $2(33.3)$ & $2(20.0)$ & \\
\hline \multicolumn{5}{|l|}{ Rete testis invasion $(n, \%)$} \\
\hline Yes & $1(25.0)$ & $1(16.7)$ & $2(20.0)$ & \multirow{2}{*}{$\S 0.667$} \\
\hline No & $3(75.0)$ & $5(83.3)$ & $8(80.0)$ & \\
\hline \multicolumn{5}{|l|}{ Tumor diameter $>4 \mathrm{~cm}(\mathrm{n}, \%)$} \\
\hline Yes & $3(75.0)$ & $4(66.7)$ & $7(70.0)$ & \multirow{2}{*}{$\S 0.667$} \\
\hline No & $1(25.0)$ & $2(33.3)$ & $3(30.0)$ & \\
\hline \multicolumn{5}{|c|}{ Lymphovascular invasion $(n, \%)$} \\
\hline Yes & $3(75.0)$ & $1(16.7)$ & $4(40.0)$ & \multirow{2}{*}{$\S 0.119$} \\
\hline No & $1(25.0)$ & $5(83.3)$ & $6(60.0)$ & \\
\hline \multicolumn{5}{|c|}{ Embryonal carcinoma rate $>50 \%(n, \%)$} \\
\hline Yes & $0(0.0)$ & $1(16.7)$ & $1(10.0)$ & \multirow{2}{*}{$\S 0.389$} \\
\hline No & $4(100.0)$ & $5(83.3)$ & $9(90.0)$ & \\
\hline \multicolumn{5}{|l|}{ Proliferation rate $>70 \%(n, \%)$} \\
\hline Yes & $0(0.0)$ & $1(16.7)$ & $1(10.0)$ & \multirow{2}{*}{$\$ 0.389$} \\
\hline No & $4(100.0)$ & $5(83.3)$ & $9(90.0)$ & \\
\hline \multicolumn{5}{|c|}{ 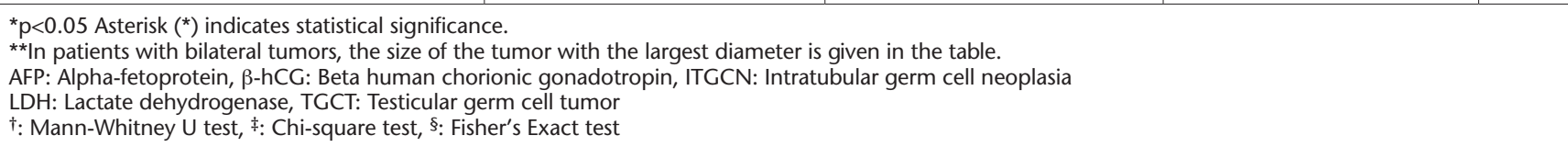 } \\
\hline
\end{tabular}

tumor in the other 5 patients. At a median follow-up of 25 (1139) months after the development of the contralateral tumor; local recurrence, distant metastasis and death were observed in one patient with stage III disease. It was noteworthy that the first tumor of this patient was also a stage III tumor. The total followup period of 6 patients with metachronous tumors after the first tumor was detected was 64 (48-69) months.
Clinical Differences Between Bilateral and Unilateral Tumors

While ITGCN ( $p=0.034)$ was found with a significantly higher rate in bilateral patients compared to unilateral patients (Table $1)$, again in these patients undescended testis $(p=0.042)$, semen parameter disorder $(p=0.030)$, hypospadias $(p=0.042)$, the incidence of testicular microlithiasis $(p=0.043)$ and TDS 
$(p=0.035)$ were higher (Table 2$)$. When we divided bilateral patients into two subgroups as patients having synchronous or metachronous tumors, no significant difference was observed between groups in terms of both ITGCN rates (Table 3 ) and other parameters mentioned (Table 4).

When the rates of local recurrence, distant metastasis and overall mortality were compared, statistical similarity was observed between both bilateral and unilateral patients, and bilateral synchronous-metachronous subgroups (Tables 2 and 4).

While NLR $(p=0.001)$, MLR $(p=0.006)$ and PLR $(p=0.013)$ were found to be significantly higher in bilateral patient; when we divided bilateral patients into subgroups within themselves, no difference was found between synchronous-metachronous subgroups (Tables 2 and 4 ).
In the median follow-up of $\mathbf{5 7 . 5}$ months for all bilateral and unilateral patients, there was no significant difference in terms of DFS, PFS and OS durations (Figure 1-3). It was observed that PFS and OS durations were significantly shorter in bilateral synchronous tumors compared to bilateral metachronous tumors (Figure 4-6).

Prognostic factors affecting contralateral tumor development in the follow-up of 68 patients with unilateral tumors at the time of diagnosis are shown in Table 5. In multivariate analysis, family history [Odds ratio (OR): 6.556, $\mathrm{p}=0.035$ )], presence of TDS (OR: 3.876, $\mathrm{p}=0.031$ ), impairment in semen parameters (OR: 2.879, $p=0.037$ ), history of undescended testis (OR: 2.561, $\mathrm{p}=0.026$ ), MLO $>0.31$ (OR: 2.234, $\mathrm{p}=0.022$ ), presence of testicular microlithiasis (OR: 2.015, $\mathrm{p}=0.015$ ), and NLR $>3.23$ (OR: $1.348, p=0.025$ ) were found to be independent variables that increased the risk of contralateral tumor development.

\begin{tabular}{|c|c|c|c|c|}
\hline Parameters & $\begin{array}{l}\text { Synchronous tumors } \\
(n=4)\end{array}$ & $\begin{array}{l}\text { Metachronous tumors } \\
(n=6)\end{array}$ & $\begin{array}{l}\text { Total } \\
(n=10)\end{array}$ & p-value \\
\hline \multicolumn{5}{|l|}{ Undescended testis (n, \%) } \\
\hline Yes & $2(50.0)$ & $2(33.3)$ & $4(40.0)$ & \multirow{2}{*}{$\S 0.548$} \\
\hline No & $2(50.0)$ & $4(66.7)$ & $6(60.0)$ & \\
\hline \multicolumn{5}{|l|}{ Semen parameter disorders (n, \%) } \\
\hline Yes & $2(50.0)$ & $2(33.3)$ & $4(40.0)$ & \multirow{2}{*}{$\S 0.548$} \\
\hline No & $2(50.0)$ & $4(66.7)$ & $6(60.0)$ & \\
\hline \multicolumn{5}{|l|}{ Hypospadias (n, \%) } \\
\hline Yes & $1(25.0)$ & $1(16.7)$ & $2(20.0)$ & \multirow{2}{*}{$\S 0.667$} \\
\hline No & $3(75.0)$ & $5(83.3)$ & $8(80.0)$ & \\
\hline \multicolumn{5}{|l|}{ Atrophic testis (n, \%) } \\
\hline Yes & $1(25.0)$ & $0(0.0)$ & $1(10.0)$ & \multirow{2}{*}{$\S 0.400$} \\
\hline No & $3(75.0)$ & $6(100.0)$ & $9(90.0)$ & \\
\hline \multicolumn{5}{|l|}{ Testicular microlithiasis (n, \%) } \\
\hline Yes & $2(50.0)$ & $3(50.0)$ & $5(50.0)$ & \multirow{2}{*}{$\S 0.738$} \\
\hline No & $2(50.0)$ & $3(50.0)$ & $5(50.0)$ & \\
\hline \multicolumn{5}{|l|}{ Presence of TDS (n, \%) } \\
\hline Yes & $2(50.0)$ & $3(50.0)$ & $5(50.0)$ & \multirow{2}{*}{$\S 0.738$} \\
\hline No & $2(50.0)$ & $3(50.0)$ & $5(50.0)$ & \\
\hline \multicolumn{5}{|l|}{ Family history of TGCT $(n, \%)$} \\
\hline Yes & $1(25.0)$ & $3(50.0)$ & $4(40.0)$ & \multirow{2}{*}{$\S 0.452$} \\
\hline No & $3(75.0)$ & $3(50.0)$ & $6(60.0)$ & \\
\hline Neutrophil/lymphocyte ratio & $6.13(4.90-7.83)$ & $4.66(3.62-7.45)$ & $5.34(4.33-7.45)$ & †0.394 \\
\hline Monocyte/lymphocyte ratio & $0.35(0.33-0.45)$ & $0.40(0.33-0.54)$ & $0.38(0.33-0.49)$ & $\dagger 0.522$ \\
\hline Platelet/lymphocyte ratio & $210.17(170.64-277.13)$ & $189.67(166.30-248.76)$ & $190.34(169.05-248.76)$ & $\dagger 0.670$ \\
\hline Mean platelet volume (fL) & $3.84(2.95-5.59)$ & $4.65(3.82-8.66)$ & $4.29(3.63-6.04)$ & $\dagger 0.286$ \\
\hline Red blood cell distribution width $(\mathrm{fL})$ & $14.75(13.80-15.10)$ & $14.55(13.32-16.40)$ & $14.75(13.57-15.37)$ & $\dagger 0.670$ \\
\hline Follow-up time [median (minimim-maximum), months] & $2(50.0)$ & $0(0.0)$ & $2(20.0)$ & $\S 0.133$ \\
\hline Local recurrence rate $(\mathrm{n}, \%)$ & $2(50.0)$ & $1(16.7)$ & $3(30.0)$ & $\S 0.333$ \\
\hline Distant metastasis rate $(n, \%)$ & $2(50.0)$ & $1(16.7)$ & $3(30.0)$ & $\S 0.333$ \\
\hline \multicolumn{5}{|c|}{$\begin{array}{l}\text { * } \mathrm{p}<0.05 \text { Asterisk }\left({ }^{*}\right) \text { indicates statistical significance. } \\
\text { TDS: Testicular dysgenesis syndrome, TCCT: Testicular germ cell tumor } \\
\dagger: \text { Mann-Whitney U test, } \neq \text { : Chi-square test, §: Fisher's Exact test }\end{array}$} \\
\hline
\end{tabular}




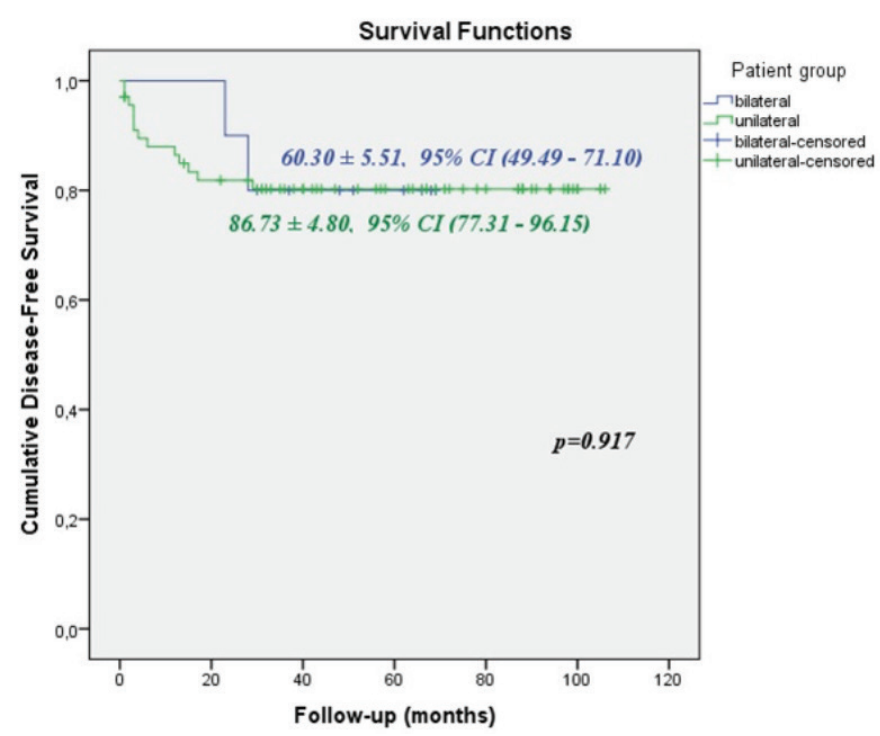

Figure 1. Plot of disease-free survival of bilateral - unilateral testicular germ cell tumors

Cl: Confidence interval

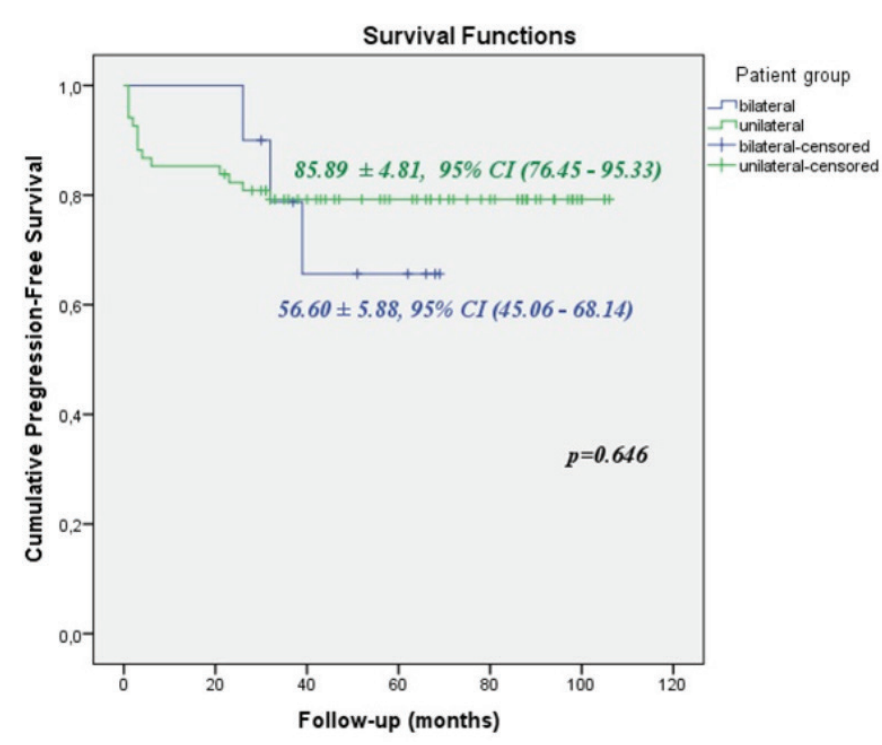

Figure 2. Plot of progression-free survival of bilateral - unilateral testicular germ cell tumors

Cl: Confidence interval

\section{Discussion}

Since bilateral TGCT was reported for the first time in 1805, patient survival rates and durations have increased thanks to the developments in adjuvant treatment strategies (7). In parallel with the increasing survival durations, a significant increase is observed especially in metachronous tumor development rates (8). Bilateral TGCT is seen at a rate of approximately $2 \%(0.5$ 7\%) (7). Of those tumors $64.7-88.9 \%$ are metachronous and 6.8-35.3\% are synchronous tumors (9). Approximately half of metachronous tumors have the same histopathology with the first tumor detected and the most common histological type is

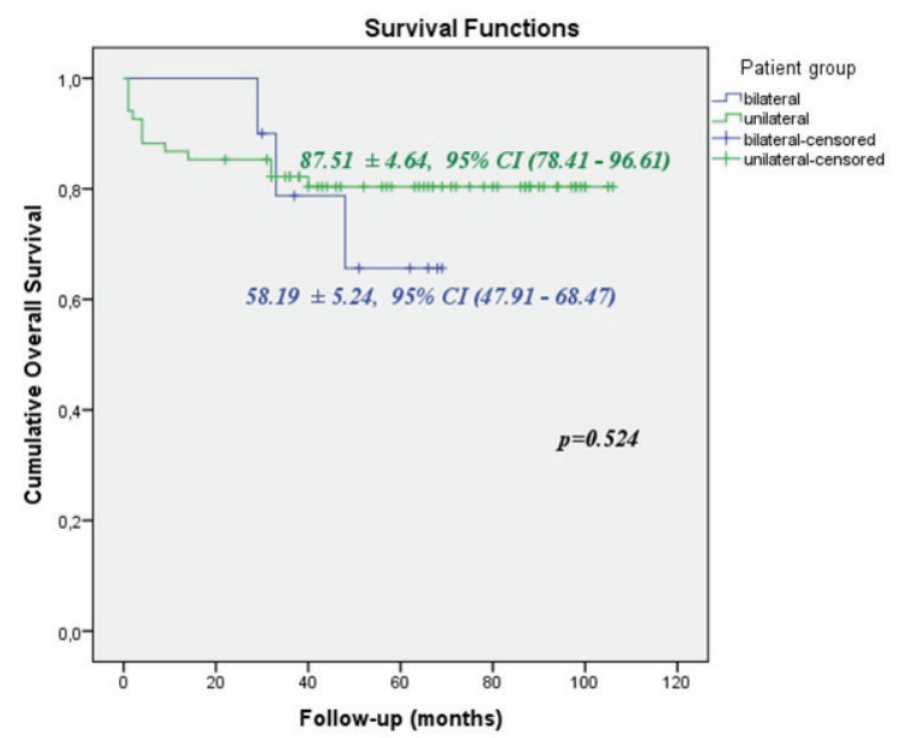

Figure 3. Plot of overall survival of bilateral - unilateral testicular germ cell tumors $\mathrm{Cl}$ : Confidence interval

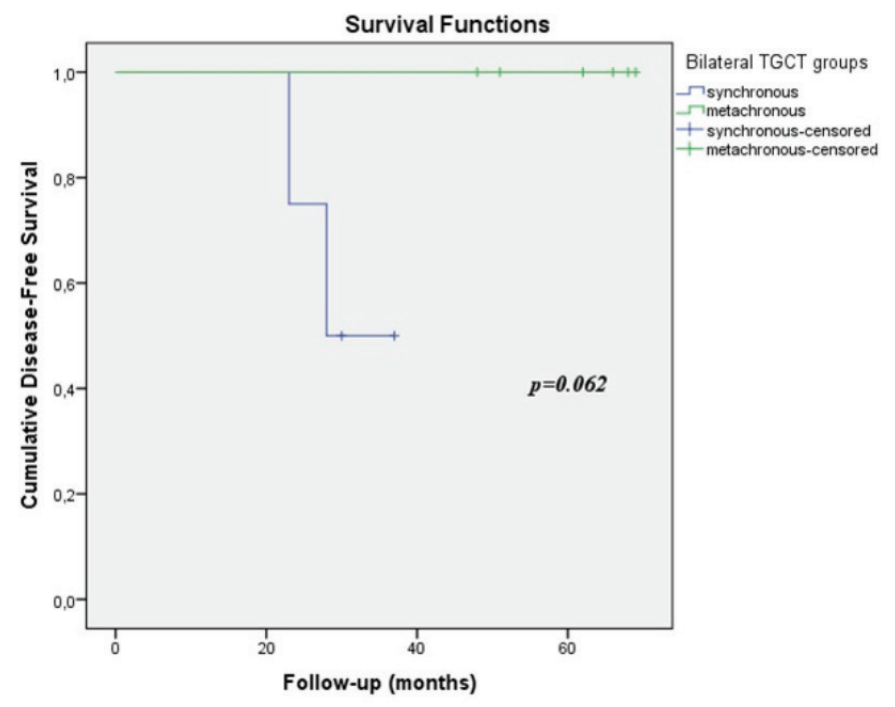

Figure 4. Plot of disease-free survival of bilateral synchronous - metachronous testicular germ cell tumors

Cl: Confidence interval, TGCT: Testicular germ cell tumours

seminoma (7). In synchronous tumors, different histopathological types are less common in both testicles and the histology seen in most of them is also seminoma (1). It has been reported that contralateral tumors develop more in patients with seminoma ( $1.8 \%$ vs $0.6 \%)$ compared to patients with non-seminomas $(10,11)$. However, in two studies conducted with limited patient data, it was stated that more contralateral tumors could develop in patients with non-seminoma than patients with seminoma $(12,13)$, and tumor histopathology was reported to have no effect on bilateral tumor development in a case series of 6 patients (14).

In our current study, although we had a small number of patients with bilateral TGCT, we found the rate of bilateral tumors to 


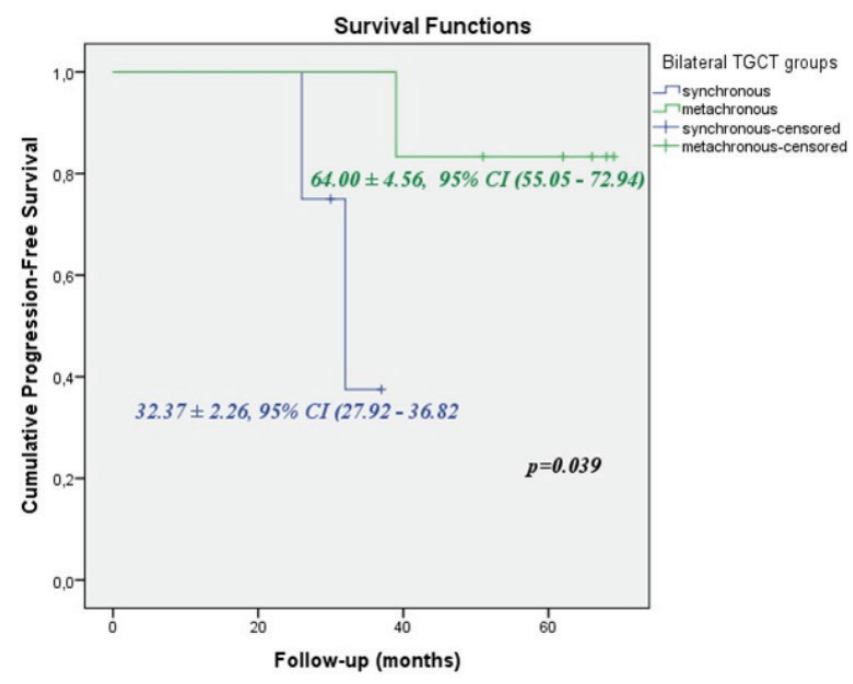

Figure 5. Plot of progression-free survival of bilateral synchronous - metachronous testicular germ cell tumors

Cl: Confidence interval, TGCT: Testicular germ cell tumours

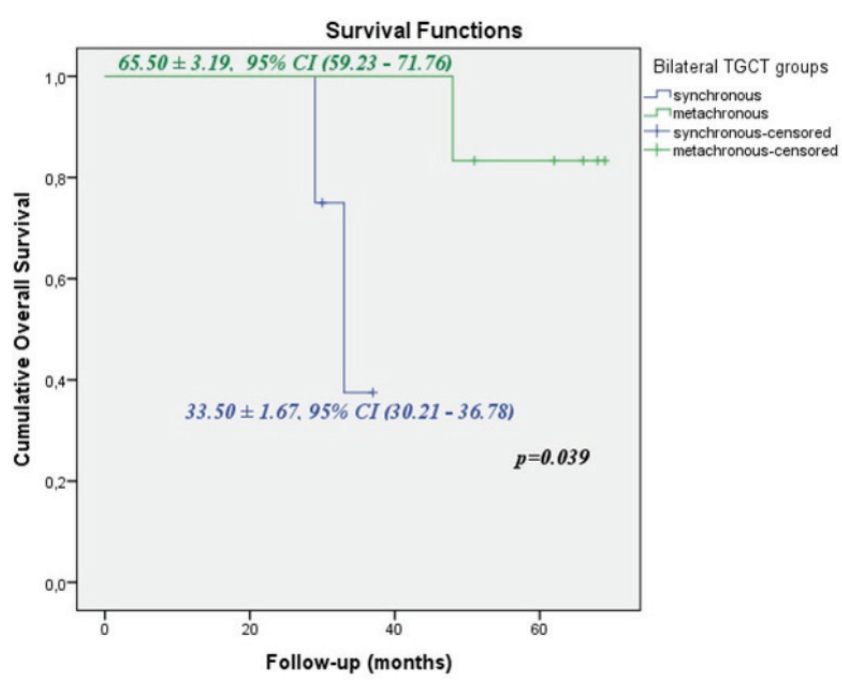

Figure 6. Plot of overall survival of bilateral synchronous - metachronous testicular germ cell tumors

Cl: Confidence interval, TGCT: Testicular germ cell tumours

\begin{tabular}{|c|c|c|c|c|c|c|c|c|}
\hline & \multicolumn{4}{|c|}{ Univariate Model } & \multicolumn{4}{|c|}{ Multivariate Model } \\
\hline & \multirow{2}{*}{ OR } & \multicolumn{2}{|c|}{ 95\% Confidence interval } & \multirow{2}{*}{$p$} & \multirow{2}{*}{ OR } & \multicolumn{2}{|c|}{$95 \%$ Confidence interval } & \multirow{2}{*}{ p } \\
\hline & & Lower & Upper & & & Lower & Upper & \\
\hline Age & 1.054 & 0.966 & 1.152 & 0.233 & & & & \\
\hline Tumor size & 1.058 & 0.823 & 1.361 & 0.659 & & & & \\
\hline Histopathologic subtype & 1.497 & 0.490 & 4.578 & 0.479 & & & & \\
\hline Tumor side (Left vs Right) & 1.910 & 0.533 & 2.381 & 0.162 & & & & \\
\hline AFP & 1.001 & 0.988 & 1.004 & 0.681 & & & & \\
\hline$\beta$-hCG & 1.012 & 0.895 & 1.058 & 0.828 & & & & \\
\hline LDH & 1.014 & 0.912 & 1.095 & 0.835 & & & & \\
\hline Tumor stage & 1.128 & 0.440 & 2.890 & 0.801 & & & & \\
\hline ITGCN (Yes vs No) & 2.533 & 0.434 & 14.778 & $0.032^{*}$ & & & & \\
\hline Rete testis invasion (Yes vs No) & 1.538 & 0.167 & 14.084 & 0.704 & & & & \\
\hline Lymphovascular invasion (Yes vs No) & 2.557 & 0.281 & 23.255 & 0.404 & & & & \\
\hline Embryonal carcinoma rate $>50 \%$ & 1.798 & 0.196 & 16.393 & 0.603 & & & & \\
\hline Proliferation rate $>70 \%$ & 3.400 & 0.725 & 7.694 & 0.081 & & & & \\
\hline Undescended testis (Yes vs No) & 3.750 & 0.589 & 23.867 & $0.042^{*}$ & 2.561 & 1.243 & 13.456 & $0.026^{*}$ \\
\hline Semen parameter disorders (Yes vs No) & 4.357 & 0.672 & 28.240 & $0.023^{*}$ & 2.879 & 1.457 & 15.457 & $0.037^{*}$ \\
\hline Hypospadias (Yes vs No) & 2.400 & 0.725 & 7.694 & $0.041^{*}$ & & & & \\
\hline Atrophic testis (Yes vs No) & 1.001 & 0.456 & 1.965 & 0.789 & & & & \\
\hline Testicular microlithiasis (Yes vs No) & 3.857 & 0.701 & 21.216 & $0.021^{*}$ & 2.015 & 1.127 & 9.420 & $0.015^{*}$ \\
\hline Testicular Dysgenesis Syndrome (Yes vs No) & 5.182 & 0.923 & 29.100 & $0.042^{*}$ & 3.876 & 1.523 & 10.123 & $0.031^{*}$ \\
\hline Family history of TGCT (Yes vs. No) & 6.556 & 1.142 & 37.621 & $0.035^{*}$ & 6.556 & 1.142 & 37.621 & $0.035^{*}$ \\
\hline $\mathrm{NLR}>3.23$ & 1.433 & 1.002 & 2.052 & $0.041^{*}$ & 1.348 & 0.859 & 3.145 & $0.025^{*}$ \\
\hline MLR $>0.31$ & 4.312 & 2.091 & 14.177 & $0.027^{*}$ & 2.234 & 1.158 & 7.815 & $0.022^{*}$ \\
\hline PLR $>150.01$ & 1.101 & 0.595 & 2.017 & $0.044^{*}$ & & & & \\
\hline $\mathrm{MPV}>5.08$ & 1.084 & 0.847 & 1.386 & 0.522 & & & & \\
\hline RDW $>13.90$ & 1.282 & 0.867 & 1.896 & 0.213 & & & & \\
\hline \multicolumn{9}{|c|}{$\begin{array}{l}\text { *p<0.05 Asterisk }\left({ }^{*}\right) \text { indicates statistical significance. } \\
\text { AFP: Alpha-fetoprotein, } \beta \text {-hCG: Beta human chorionic gonadotropin, ITGCN: Intratubular germ cell neoplasia } \\
\text { LDH: Lactate dehydrogenase, MLO: Monocyte/lymphocyte ratio, MPV: Mean platelet volume, } \\
\text { NLR: Neutrophil/lymphocyte ratio, PLR: Platelet/lymphocyte ratio, RDW: Red blood cell distribution width, } \\
\text { TGCT: Testicular germ cell tumor, OR: Odds ratio }\end{array}$} \\
\hline
\end{tabular}


be $11.4 \%$, above the rates reported in the literature, since we were a selected center where oncology patients were referred. Consistent with the literature, $40 \%$ of these patients had synchronous and $60 \%$ had metachronous tumors. While pure seminoma was present on both sides of all synchronous tumors; in $2(33.3 \%)$ patients with metachronous tumors, contralateral tumor was observed to be seminoma, unlike the first nonseminoma tumor. When we examined a total of 68 patients with unilateral tumors at the time of diagnosis, contrary to most publications reported in the literature and similar to the studies of Osterlind et al. (13) and Colls et al. (12); we found that more contralateral metachronous tumors developed in patients with non-seminoma (10.8\% vs $5.4 \%$ ) than patients with seminoma.

Survival and remission rates in bilateral patients were found to be similar to unilateral patients in some studies (7). When bilateral patients were compared within themselves, it was observed that the clinical stage was higher in synchronous tumors, and the DFS and OS rates were found to be lower (1). It was observed that DFS and OS rates of metachronous tumors decreased when he clinical stage was higher, when the time until the second tumor development was $>60$ months, and when the histopathology of the first tumor was seminoma (1). Unlike these findings, Holzbeierlein et al. (15) observed that most of the bilateral synchronous and metachronous tumors were at low stage. Klatte et al. (16), on the other hand, reported that, although bilateral synchronous tumors were diagnosed at a higher stage, if an effective treatment was applied, oncologically similar results could be obtained in synchronous and metachronous tumors compared with unilateral tumors. We also observed similar rates among bilateral and unilateral patients and between bilateral synchronous and bilateral metachronous subgroups in terms of local recurrence, distant metastasis and overall mortality rates in our patients. However, we found that the PFS and OS durations were significantly shorter in bilateral synchronous tumors than in bilateral metachronous tumors, and that stage III disease developed more in synchronous tumors (75\% vs $33.3 \%$ ).

According to the common interpretation of most studies in the literature; since seminomas are thought to be less aggressive than non-seminomas, active surveillance is used more frequently than adjuvant therapy in patients with bilateral synchronous seminomas. It has been suggested that this may be the reason for the greater decrease in DFS and OS durations of synchronous seminomas compared to synchronous nonseminomas $(1,17,18)$. A similar decrease in survival rates was observed in patients with metachronous tumors in whom seminoma developed in the contralateral testicle after the first seminoma compared to metachronous tumors with nonseminoma histopathology, and this situation was attributed to the same cause (1). Zequi et al. (1) observed that seminoma developed at a higher rate in patients who developed tumor in the contralateral testis during a follow-up period longer than 60 months. Depending on the above-mentioned interpretation, they stated that the DFS and OS rates decreased in relation to this situation, since more seminomas were seen when the time until the second tumor development was $>60$ months. As is known in most solid tumors, the prolongation of the time between the onset of the primary lesion and the recurrence or metastasis is a good prognostic indicator. However, metachronous testicular tumors were not metastases, and according to Zequi et al.'s argument (1), the prolongation of the time until the detection of metachronous tumors increased the likelihood of development of seminoma and, on the contrary, it was found to be associated with a poor prognosis. Nevertheless, there is no accepted clear consensus to explain this situation and both the findings of Holzbeierlein et al. (15) and Klatte et al. (16) and our findings do not support the mentioned relationship.

It was found that the frequency of metachronous tumor development was lower in patients who underwent adjuvant CT. In addition, it was observed that more seminoma was observed in metachronous tumors developing in patients who underwent CT compared to metachronous tumors developing in the follow-up of patients who did not undergo $C T$, regardless of the initial tumor histology (1). Although there were publications predicting that adjuvant $\mathrm{CT}$ would reduce the risk of contralateral tumor development $(16,19)$, there were also studies in which this relationship was not observed $(1,20)$. We realized that adjuvant radiotherapy (RT) was applied to patients who were diagnosed as having stage I seminoma and were treated between 2010 and 2013 in our study, as part of the treatment protocol of that period. However, adjuvant RT has been replaced by single-dose carboplatine in the treatment of these patients in the EAU guidelines since 2014 (3). Of our 68 patients with unilateral tumors at the time of diagnosis, contralateral tumors were observed in $20 \%$ of those who were under active follow-up because they had stage I disease and did not have a risk factor for occult metastasis development, while the rate of contralateral tumor development after adjuvant CT or RT was lower (6.25\%). In our 2 patients who developed contralateral tumors after active follow-up, we observed that the rate of development of seminoma was higher, consistent with the findings of Zequi et al. (1).

While the incidence of TGCT development is $0.005 \%$ in the general population, the rate of tumor development in the contralateral testicle in individuals with a history of TGCT is up to $5 \%$ (1). Despite this increased risk, it was observed that the tumor detected in the contralateral testis in patients with metachronous tumors was mostly (95.2\%) stage I tumor and the developing second tumor was smaller than the first tumor $(10,11)$. Therefore, it has been emphasized that self-examination and early diagnosis are important in patients with a history of TGCT (10). The time until the second tumor development has been reported as a median of $39-47$ months (4 months-32 years) in different studies $(1,7,16,17)$. Among our 68 patients with unilateral tumors at the time of diagnosis, contralateral tumors were detected in $8.1 \%$ of them during a follow-up of a median 57.5 months, and a second tumor emerged within 5 years, after the first tumor was detected in all metachronous tumors. But we did not observe a significant difference in the size of the second tumors in our patients.

ITGCN is defined as a precursor lesion in the development of TGCT (1). There are still controversies about the application of biopsy to confirm the presence of ITGCN in the contralateral testis during orchiectomy, since the incidence of ITGCN and tumor development in the contralateral testis is low, the developing metachronous tumors are mostly at low stage, and side effects such as infertility and testosterone production 
disorders due to local RT to be applied in case of detection of ITGCN, can be observed $(16,21)$. However, it is known that the risk of developing ITGCN in the contralateral testis is $>35 \%$ in patients with unilateral tumors under the age of 40 with testicular volumes of $<12 \mathrm{~mL}(22)$. Therefore, contralateral testis biopsy is recommended during orchiectomy in patients under 40 years of age with risk factors for the development of TGCT (testicular volume $<12 \mathrm{~mL}$, history of undescended testis or impaired spermatogenesis) (3). In our study, ITGCN was detected in 34 of 68 patients who had unilateral tumors at the time of diagnosis, and 15 of these 68 patients (20.2\%) who had the above risk factors did not undergo contralateral testicular biopsy, because none of them accepted it. We observed that 4 (26.6\%) of 15 patients with risk factors developed contralateral metachronous tumors. Of these 4 patients, 2 (50\%) had a history of undescended testis, 2 (50\%) had abnormal semen parameters, while all were under the age of 40 , but none of them had atrophic testis. Since our findings are consistent with the data in the literature, we continue to present testicular biopsy as a recommendation to patients with these risks in our clinical practice.

Although the gold standard approach is bilateral orchiectomy in cases of bilateral TGCT, the most important problems related to this are infertility, the need for lifelong androgen maintenance, and the psychological effects observed due to the young age of the patients $(7,10)$. Therefore, if preoperative serum testosterone levels are normal in small-sized $(<2 \mathrm{~cm}$ in size or tumor volume is less than $30 \%$ of testicular volume) synchronous bilateral and metachronous contralateral tumors, testis-sparing surgery (partial orchiectomy) can be used as an alternative approach (3). However, if ITGCN is detected histologically in the remaining testicular tissue, it is recommended to give adjuvant RT to the testis on that side (3). Therefore, patients who want to have children should be given detailed information about this situation. Partial orchiectomy was not performed in any patient among 10 patients with bilateral tumors in our study considering that there would not be enough testicular parenchyma to be preserved, because tumor size was not less than $2 \mathrm{~cm}$ or the estimated tumor volume/testicular volume ratio was not less than $30 \%$.

Individuals with a family history are at higher risk for developing TGCT, and the rate of bilateral tumor development is higher in these individuals compared to sporadic patients (10). In our patients with bilateral tumors, family history was significantly higher than in patients with unilateral tumors (40\% vs $13.2 \%$ ). When bilateral tumors were examined within themselves, we found that although no significant difference was found between synchronous and metachronous tumors in terms of family history, family history was the risk factor that increased tumor development 6.5 times in the contralateral testicle.

Recently, the effects of inflammation markers and hemogram parameters on oncological outcomes have been more popularly investigated, and it has been reported that high NLR and platelet levels in TCCT may cause development of metastasis and a decrease in disease-specific survival $(23,24)$. When we examined the effects of serum hemogram parameters on our patients, it was found that NLR, MLR, PLR values at the time of diagnosis were higher in bilateral tumors compared to unilateral tumors. We found that high NLR (>3.23) and MLR (>0.31) values increased the risk of contralateral TGCT by 1.3 and 2.2 times, respectively.

The effects of molecular and genetic risk factors on the development of TGCT have not been fully elucidated yet. Recently, there are publications defending that c-kit mutations are more common in bilateral patients than unilateral patients (25), and also opposite results have been encountered $(26,27)$. Although no genetic and biomolecular markers were examined in our study, we observed that the development of TGCT in the contralateral testis significantly increased in the presence of family history, testicular microlithiasis, undescended testis with increased NLR, MLR, TDS and TDS components, and impairment in semen parameters. Although it is known that metachronous contralateral TGCT develops within the first five years in $60 \%$ of the patients, it has been reported in the literature that metachronous contralateral TGCT may develop even after 32 years and long-term follow-up is required in all patients, especially in individuals with the risk factors mentioned above (16).

\section{Study Limitations}

The retrospective design of our study, the limited power of the statistical analysis performed due to the small number of patients, lack of randomization while determining the groups, short follow-up periods, and the fact that the follow-up results belonging to a single center, were the main limiting factors. In addition, since our hospital was a center where oncology patients were referred, the patients we included in the study mostly represented a selected group with advanced stage disease. For this reason, bilateral TGCT detection, recurrence, progression and death rates in our study were higher than the rates reported in the literature. We think that these rates may not reflect the real population incidence.

\section{Conclusion}

According to our findings, although bilateral synchronous tumors were detected at a more advanced stage at the time of diagnosis, we showed that the survival rates of bilateral tumors were similar to unilateral tumors thanks to appropriate treatments, although a significant decrease in PFS and OS times was observed. Nevertheless, we observed that patients with unilateral tumors at the time of diagnosis increased the risk of development of contralateral tumors in cases of family history, TDS, impaired semen parameters, undescended testicular history, presence of testicular microlithiasis, NLR $>3.23$ and MLR $>0.31$. If patients with a history of TGCT carry these risk factors, it is important to follow-up the contralateral testis regularly, as the possibility of development of bilateral metachronous tumors in long-term follow-up increases.

\section{Acknowledgements}

Publication: The results of the study were not published in full or in part in form of abstracts.

Contribution: There is not any contributors who may not be listed as authors. 
Conflict of Interest: No conflict of interest was declared by the authors.

Financial Disclosure: The authors declared that this study received no financial support.

\section{Ethics}

Ethics Committee Approval: All procedures in our study were conducted in accordance with the ethical standards of the institutional and national research committee including human participants and the principles of the Helsinki Declaration, and since it was a retrospective study, no ethics committee approval was made.

Informed Consent: Each patient was informed before the surgery that oncological follow-up information such as recurrence, metastasis development, and survival analysis can be used in various oncological studies to be performed in the clinic without specifying the patient names and identity information, and the data of patients who did not consent were not used.

Peer-review: Externally peer-reviewed.

\section{Authorship Contributions}

Surgical and Medical Practise: A.I.A., M.S.B., H.B., Concept: A.I.A., M.S.B., Design: I.S., H.B., Data Collection or Processing: I.S., Analysis or Interpretation: İ.S., A.I.A., Literature Search: I.S., Writing: I.S.

\section{References}

1. Zequi Sde C, da Costa WH, Santana TB, et al. Bilateral testicular germ cell tumours: a systematic review. BJU Int 2012;110:1102-1109.

2. McGlynn KA, Cook MB. Etiologic factors in testicular germ-cell tumors. Future Oncol 2009;5:1389-1402.

3. Laguna MP, Albers $P$, Albrecht W, et al. European Association of Urology guidelines on testicular cancer: the 2019 Update. EAU Guidelines Office, Arnhem, The Netherlands. Available: http:// uroweb.org/guidelines/compilations-of-all-guidelines/

4. Géczi L, Gomez F, Bak M, Bodrogi I. The incidence, prognosis, clinical and histological characteristics, treatment, and outcome of patients with bilateral germ cell testicular cancer in Hungary. J Cancer Res Clin Oncol 2003;129:309-315.

5. Fosså SD, Chen J, Schonfeld SI, et al. Risk of contralateral testicular cancer: a population-based study of 29,515 U.S. men. J Natl Cancer Inst 2005;97:1056-1066.

6. van den Driesche S, Kilcoyne KR, Wagner I, et al. Experimentally induced testicular dysgenesis syndrome originates in the masculinization programming window. JCI Insight 2017;2:e91204.

7. Morales-Barrera R, Valverde C, Rodón J, et al. Bilateral testicular germ cell tumours: a single hospital experience. Clin Transl Oncol 2010;12:299-302.

8. McGlynn KA, Devesa SS, Sigurdson AJ, et al. Trends in the incidence of testicular germ cell tumors in the United States. Cancer 2003;97:63-70.

9. Che M, Tamboli P, Ro JY, et al. Bilateral testicular germ cell tumors: twenty year experience at M. D. Anderson Cancer Center. Cancer 2002;95:1228-1233.
10. Akdogan B, Divrik RT, Tombul T, et al. Bilateral testicular germ cell tumors in Turkey: increase in incidence in last decade and evaluation of risk factors in 30 patients. J Urol 2007;178:129-133.

11. Sun BL, Pearl R, Sharifi R, Guzman G. Metachronous bilateral testicular seminoma developing after an interval of 31 years: case report and review of the literature. Int J Surg Pathol 2015;23:156-160.

12. Colls BM, Harvey VI, Skelton L, et al. Bilateral germ cell testicular tumors in New Zealand: experience in Auckland and Christchurch 1978-1994. J Clin Oncol 1996;14:2061-2065.

13. Osterlind A, Berthelsen JG, Abildgaard N, et al. Risk of bilateral testicular germ cell cancer in Denmark: 1960-1984. J Natl Cancer Inst 1991;83:1391-1395.

14. Tabernero J, Paz-Ares L, Salazar R, et al. Incidence of contralateral germ cell testicular tumors in South Europe: report of the experience at 2 Spanish university hospitals and review of the literature. J Urol 2004;171:164-167.

15. Holzbeierlein JM, Sogani PC, Sheinfeld J. Histology and clinical outcomes in patients with bilateral testicular germ cell tumors: the Memorial Sloan Kettering Cancer Center experience 1950 to 2001. J Urol 2003;169:2122-2125.

16. Klatte $T$, de Martino $M$, Arensmeier $K$, et al. Management and outcome of bilateral testicular germ cell tumors: a 25-year single center experience. Int J Urol 2008;15:821-826.

17. Kopp RP, Chevinsky M, Bernstein M, et al. Bilateral Testicular Germ Cell Tumors in the Era of Multimodal Therapy. Urology 2017; 103:154-160.

18. Schaapveld M, van den Belt-Dusebout AW, Gietema JA, et al. Risk and prognostic significance of metachronous contralateral testicular germ cell tumours. Br J Cancer 2012;107:1637-1643.

19. Sokoloff MH, Joyce GF, Wise M. Testis cancer. J Urol 2007;177: 2030-2041.

20. Pamenter B, De Bono JS, Brown IL, et al. Bilateral testicular cancer: a preventable problem? Experience from a large cancer center. BJU Int 2003;92:43-46.

21. Harland SJ, Cook PA, Fosså SD, et al. Intratubular germ cell neoplasia of the contralateral testis in testicular cancer: defining a high risk group. J Urol 1998;160:1353-1357.

22. Krege S, Beyer J, Souchon R, et al. European Consensus Conference on Diagnosis and Treatment of Germ Cell Cancer: A report of the second meeting of the European Germ Cell Cancer Consensus Group (EGCCCG): Part II. Eur Urol 2008;53:497-513.

23. Bolat D, Aydoğdu Ö, Polat S, et al. Predictive value of preoperative neutrophil-to-lymphocyte ratio on the prognosis of germ cell testicular tumors. Turk J Urol 2017;43:55-61.

24. Koca O, Kutluhan MA, Akyüz M, et al. Hematological evolution of patients with testicular cancer. New J Urol 2017;12:32-35.

25. Looijenga $L H$, de Leeuw $H$, van Oorschot $M$, et al. Stem cell factor receptor (c-KIT) codon 816 mutations predict development of bilateral testicular germ-cell tumors. Cancer Res 2003;63:7674-7678.

26. Sakuma Y, Matsukuma S, Yoshihara M, et al. Mutations of c-kit gene in bilateral testicular germ cell tumors in Japan. Cancer Lett 2008;259:119-126.

27. Coffey J, Linger R, Pugh J, et al. Somatic KIT mutations occur predominantly in seminoma germ cell tumors and are not predictive of bilateral disease: report of 220 tumors and review of literature. Genes Chromosomes Cancer 2008;47:34-42. 